\title{
The effects of body weight and pneumoperitoneum on Pleth Variability Index and Total Hemoglobin in patients undergoing laparoscopic cholecystectomy: a prospective observational study.
}

\author{
Gulcin Patmano ${ }^{1}$, mehmet patmano ${ }^{2}$, tufan gümüş ${ }^{3}$, and mikail kılınç ${ }^{4}$ \\ ${ }^{1}$ Sanliurfa Mehmet Akif Inan Training and Research Hospital \\ ${ }^{2}$ Kayseri City Education and Research Hospital \\ ${ }^{3}$ Ege Universitesi \\ ${ }^{4}$ mediline hospital elazı̆
}

June 19, 2021

\begin{abstract}
Abstract Background It is not clear if there is a inaccurate effect of obesity and pneumoperitoneum on non-invasive monitoring parameters Objectives To determine the effects of $\mathrm{CO} 2$ pneumoperitoneum and desufflation on non-invasive monitoring variables between obese and non-obese patients undergoing laparoscopic surgery. Methods Sixty patients were included in the study who underwent laparoscopic cholecystectomy between February 2019-November 2019. After anesthesia induction, systolic and diastolic blood pressures, heart rate(HR), operation and pneumoperitoneum durations, PVI and SpHg data were recorded. Results The patients were divided into two groups. There was no statistically significant difference between the groups in terms of chronic diseases, operation and pneumoperitoneum durations. Statistically no significant difference was found between the groups for PVI and SpHb values. In comparison of PVI measurement values according to T0, a significant difference was found in T3,T4 and T5 in Group 2 compared to T0 ( $\mathrm{p}=0.010 ; 0.012 ; 0.041)$. In comparison of SpHb measurement values according to T0, a statistically significant difference was found in T1,T2 and T3 in Group 2 compared to T0 ( $\mathrm{p}=0.008 ; 0.010 ; 0.037)$. In comparison to T5, there was a significant difference in T1,T2 and T3 in Group 2 ( $\mathrm{p}=0.023 ; 0.005 ; 0.006)$. Conclusion In obese people, pneumoperitonium can lead to inaccuracies in monitoring PVI and SpHb. Although these two parameters are very valuable in intraoperative follow-up, they should be used carefully during laparoscopic procedures in obese patients. If these parameters (PVI and $\mathrm{SpHb}$ ) are considered to be used in bariatric surgeries, this information should be considered.
\end{abstract}

The effects of body weight and pneumoperitoneum on Pleth Variability Index and Total Hemoglobin in patients undergoing laparoscopic cholecystectomy: a prospective observational study.

\section{Abstract \\ Background}

It is not clear if there is a inaccurate effect of obesity and pneumoperitoneum on non-invasive monitoring parameters

\section{Objectives}

To determine the effects of $\mathrm{CO} 2$ pneumoperitoneum and desufflation on non-invasive monitoring variables between obese and non-obese patients undergoing laparoscopic surgery.

\section{Methods}


Sixty patients were included in the study who underwent laparoscopic cholecystectomy between February 2019-November 2019. After anesthesia induction, systolic and diastolic blood pressures, heart rate(HR), operation and pneumoperitoneum durations, PVI and SpHg data were recorded.

\section{Results}

The patients were divided into two groups. There was no statistically significant difference between the groups in terms of chronic diseases, operation and pneumoperitoneum durations. Statistically no significant difference was found between the groups for PVI and SpHb values. In comparison of PVI measurement values according to T0, a significant difference was found in T3,T4 and T5 in Group 2 compared to T0 $(\mathrm{p}=0.010 ; 0.012 ; 0.041)$. In comparison of $\mathrm{SpHb}$ measurement values according to $\mathrm{T} 0$, a statistically significant difference was found in T1,T2 and T3 in Group 2 compared to T0 ( $\mathrm{p}=0.008 ; 0.010 ; 0.037)$. In comparison to $\mathrm{T} 5$, there was a significant difference in T1,T2 and T3 in Group $2(\mathrm{p}=0.023 ; 0.005 ; 0.006)$.

\section{Conclusion}

In obese people, pneumoperitonium can lead to inaccuracies in monitoring PVI and SpHb. Although these two parameters are very valuable in intraoperative follow-up, they should be used carefully during laparoscopic procedures in obese patients. If these parameters (PVI and $\mathrm{SpHb}$ ) are considered to be used in bariatric surgeries, this information should be considered.

Key words : Laparoscopic cholecystectomy; pneumoperitoneum; pleth variability index; total hemoglobin; obesity

One of the dynamic parameters used to predict fluid response in mechanically ventilated patients is the platelet variability index (PVI), which is obtained by continuous and automatic calculation of pulse oximetry plethysmographic waveform variability during the respiratory cycle. Factors such as arrhythmia, hypothermia, spontaneous respiratory activity, vasoactive drug use, and impaired peripheral perfusion limit the presence of PVI and SpHb monitoring. The ability of these dynamic parameters to predict fluid response and blood loss may be affected by respiratory, hemodynamic, and physical changes that cause changes in intrathoracic pressure.

In the obese, pneumoperitoneum can lead to errors in PVI and SpHb monitoring. Although these two parameters are very valuable in intraoperative follow-up, they should be used with caution during laparoscopic procedures in obese patients. This information should be considered if these parameters (PVI and $\mathrm{SpHb}$ ) are considered to be used in bariatric surgeries, which is the most frequently performed surgical procedure with the combination of obesity and pneumoperitoneum. This is the first study with standardized patient groups on the reliability of PVI and SpHb in obesity and pneumoperitoneum.

\section{Introduction}

With the development of mini-invasive technique, laparoscopic procedures form the basis of general surgery. After the introduction of laparoscopy in surgery, laparoscopic cholecystectomy has been adopted as the gold standard in the surgical treatment of cholelithiasis and gallbladder diseases (1). "CO2 pneumoperitonium method" is used in exposing sufficient image and surgical field in laparoscopy. Usually, artificial pneumoperitoneum is established by carbon dioxide insufflation and intra-abdominal pressure (IAP) is often kept between 10 and $15 \mathrm{mmHg}$.

There are many advantages of laparoscopic cholecystectomy (such as short hospital stay, minimal postoperative pain, rapid recovery), as well as systemic disadvantages associated with increased intraabdominal pressure. In laparoscopic cholecystectomy procedures, cardiopulmonary changes may be observed due to the pneumoperitoneum (2). Since preload is significantly affected by IAP increase, it is important to determine the effect of IAP increase on fluid response in patients undergoing laparoscopic surgery (3).

Today, hemodynamic monitoring includes static parameters such as pulmonary capillary wedge pressure, central venous pressure, as well as dynamic parameters such as pulse pressure variation and stroke volüme 
variation (4). One of the dynamic parameters used to predict fluid response in mechanically ventilated patients is the pleth variability index (PVI) obtained by continuous and automatic calculation of pulse oximetry plethysmographic waveform variability during the respiratory cycle. The Masimo Radical-7 (Masimo Corporation, Irvine, CA) device provides an easy and continuous measurement using a finger probe. This device has been validated to predict fluid sensitivity in patients who are mechanically ventilated and undergo open abdominal surgery $(5,6)$. A continuous, non-invasive Hb level monitoring is provided simultaneously with the same probe. This has also been shown to correlate with blood hg levels (7). As this device can be used non-invasively, it has become the preferred hemodynamic monitoring method for patient follow-up.

However, factors such as arrhythmia, hypothermia, spontaneous breathing activity, vasoactive drug use, and impaired peripheral perfusion limit the availability of PVI and SpHb monitoring (8). The ability of these dynamic parameters to predict fluid response and blood loss may be affected by respiratory, hemodynamic and physical changes that cause intrathoracic pressure change (9).

There are few studies examining the effect of pneumoperitoneum or obesity on the reliability of these monitors. Both factors have previously been shown to significantly affect cardiopulmonary physiology and function, which both potentially affect the performance and reliability of these devices (10). There are studies investigating the effects of both pneumoperitoneum and obesity in gastric bypass operations, but there is no study investigating the effects of pneumoperitoneum with equal intraabdominal pressures, in obese and non-obese patients (11). The aim of this study was to determine the effects of continuous CO2 pneumoperitoneum with a pressure of $12 \mathrm{mmHg}$ and desufflation on non-invasive monitoring variables between obese and non-obese patients undergoing laparoscopic surgery.

\section{Methods}

\section{Subjects}

This study was carried out in accordance with the Declaration of Helsinki and approved by the Ethics Committee of the Harran University (Approval Number 2019.4.20). After the approval of the hospital ethics committee, The study was registered in the Australian New Zealand Clinical Trials Registry (ANZCTR) (Trial Id: ACTRN12620000169943). Patients who underwent elective laparoscopic cholecystectomy under general anesthesia for cholelithiasis between November 2019 and February 2019 were included in this cross sectional study. Written informed consent was obtained from all patients. Patients over the age of 18 years with written consent and with an American Anesthesiologists Association (ASA) physical score I or II were included in the study. Exclusion criteria; Pregnancy, acute lung disease, heart failure, body mass index (BMI) below 18 and above 40, intraoperative bleeding over $100 \mathrm{ml}$, intraoperative inotropic support requirement, pneumoperitoneum duration over $45 \mathrm{~min}$ and under $20 \mathrm{~min}$. All patients were fasted for at least 8 hours before surgery, and none of the patients received premedication.

Anesthesia management and hemodynamic monitoringBefore general anesthesia induction, standard anesthetic monitoring was performed with electrocardiography (ECG), peripheral O2 saturation (SpO2), non-invasive arterial pressure (NIBP) monitoring. The MightySat Rx Fingertip Pulse Oximeter (Masimo Corporation, Irvine, CA) probe was attached preventing it from being affected by light to the upper extremity index finger tip, where the blood pressure cuff is not attached. Anesthesia induction was performed by the same anesthetist with fentanyl $(2 \mu \mathrm{g} / \mathrm{kg})$ and propofol $(2-3 \mathrm{mg} / \mathrm{kg})$. Rocuronium bromide $0.6 \mathrm{mg} / \mathrm{kg}$ was used as a muscle relaxant. Volume controlled ventilation was applied to the patients after intubation. Ventilator settings were made as follows: tidal volume was $8 \mathrm{ml} / \mathrm{kg}$ according to ideal body weight, positive end-tidal pressure (PEEP) was $5 \mathrm{cmH} 2 \mathrm{O}$, inspirium expirium ratio was 1: 2, respiratory rate was 11-14 breaths / minute and then the end-tidal carbon dioxide pressure (EtCO2) was adjusted to be 35-45 $\mathrm{mmHg}$. Sevoflurane and iv remifentanil $(0.25-0.5 \mu \mathrm{g} / \mathrm{kg} / \mathrm{min})$ infusion was used in the mixture of air and oxygen for maintenance of anesthesia.

Study design Data were collected after anesthesia induction in all patients. In order to minimize the effect of vasomotor tone, any stimulation to the patients was avoided up to 5 minutes before the data was collected. PVI and SpHg data were recorded by the same anesthesiologist in 6 pre-determined times in 
the end-expiratory phase. $\mathrm{T} 0=$ basal value after insufflation $\mathrm{T} 1=5$ th minute of insufflation $\mathrm{T} 2$ : 10 th minute of insufflation T3: 15 th minute of insufflation T4 $=5$ minutes before desufflation $\mathrm{T} 5=2$ minutes after desufflation Systolic blood pressure (SBP), diastolic blood pressure (DBP), heart rate (HR), oxygen saturation $(\mathrm{SpO} 2), \mathrm{EtCO} 2$ values were recorded in the same time frames. In addition, body mass index, operation duration and pneumoperitoneum duration of the patients were recorded. $8 \mathrm{ml} / \mathrm{kg}$ crystalloid solution was given to patients before anesthesia induction in order to prevent sudden blood pressure decrease that may develop in patients as a result of anesthesia induction, thus minimizing the impact of PVI and $\mathrm{SpHb}$ values. Crystalloid infusion continued at the rate of $4 \mathrm{ml} / \mathrm{kg} / \mathrm{h}$ throughout the operation.

\section{Statistical analysis}

All statistical analyses were calculated by SPSS 22.0 (Statistical Packages for the Social Sciences, SPPS Inc., Chicago, IL, USA). The normal distribution was determined by Kolmogorov Smirnov test and histogram. All of the parameters were not normally distributed probably because of the low group sizes. Non-parametric tests are used for calculations. The continuous variables were expressed as median (min-max). The categorical variables were expressed as n (\%). The differences of continuous variables were calculated by the Mann Whitney-U test and the Wilcoxon signed rank test was used for repeated measures. Chi-Square test was used to determine the difference between groups of categorical variables. $\mathrm{p}<0.05$ were considered as statistically significant. The sample size was determined using the preliminary data of 10 patients. In power analysis, a minimum of 48 patients were shown for $\beta=0.1$ and $\alpha=0.05$.

Results After obtaining the ethics committee approval, 74 patients were included in the study. Out of 74 patients, 60 patients were included in this study, after exclusion of 14 patients according to the exclusion criteria (Figure 1). Thirty patients with BMI $<30$ were classified as Group 1, the remaining 30 patients with BMI [?] 30 were classified as Group 2. The mean age of the patients was $38,22 \pm 10,35$ and $61,6 \%$ of the patients were women. There was no statistically significant difference between the groups in terms of chronic diseases, operation duration and pneumoperitoneum duration. Demographic data of the patients are shown in Table 1. Data on systolic blood pressure, diastolic blood pressure and heart rates are shown in Figures 2 and 3. From these data, only the systolic blood pressure baseline value was significantly higher in Group $2(\mathrm{p}=0.007)$, but there was no significant difference between the groups in other time frames and other measurements. The comparison of hemodynamic monitoring parameters between T0 and T5 is shown in Table 2. Statistically no significant difference was found between the groups at all measurement times for PVI and SpHb values. Figure 4 and Figure 5 show the changes of PVI and SpHb over time. Measurements over all time periods were compared with T0 and T5 separately. In comparison of PVI measurement values with T0, a statistically significant difference was found in T3, T4 and T5 in Group 2 ( $\mathrm{p}=0.010 ; 0.012 ; 0.041$, respectively). In comparison with $\mathrm{T} 5$, statistically no significant difference was found in both groups. In comparison of $\mathrm{SpHb}$ measurement values with $\mathrm{T} 0$, a statistically significant difference was found in $\mathrm{T} 1, \mathrm{~T} 2$ and T3 in Group 2 ( $\mathrm{p}=0.008 ; 0.010 ; 0.037$, respectively). In comparison with T5, there was a statistically significant difference in T1, T2 and T3 in Group 2 ( $\mathrm{p}=0.023 ; 0.005 ; 0.006$, respectively).

\section{Discussion}

The main finding of our study was, there was a false increase in PVI and a false decrease in SpHb with abdominal insufflation. Although this situation developed in all patients, it was found statistically significant only in the obese patient group (Group 2). Also, in obese, SpHb was affected by desufflation as well as insufflation.

As a result of advanced medical practices all over the world, the elderly population is increasing all over the world. However, elderly patients need more surgery day by day and the importance of hemodynamic monitoring is increasing at this point. Invasive procedures such as pulmonary artery catheters, central venous catheters, transesophageal echocardiogram require special training, increase complications and cost, and take time to apply $(12,13)$. Assessment of laboratory parameters such as hemoglobin $(\mathrm{Hb})$, lactate and base deficit (BE) is often used in the process of intraoperative evaluation, but operation cost also increases because of these measurements, and the evaluation of these parameters is not very effective as it takes time. 
Therefore, the use of non-invasive hemodynamic monitoring is increasingly important.

Blood loss estimation and evaluation of the intravascular volume during surgery is still a major problem. Changes in intravascular volume or airway pressures can cause changes in cardiac pumping ability (14). However, as with cardiogenic shock, not all reductions in cardiac output are associated with intravascular volume. There are also a number of recent studies regarding the poor consequences of intraoperative excess fluid overload in patients. It has been reported that the application of each liter of extra fluid added to the intraoperative fluids increases the risk of postoperative symptoms and complications by $16 \%$ and $32 \%$, respectively (15). It has been stated that patient admissions are shortened, surgical site infections are reduced and perfusion improves with sufficient but not much fluid administration $(16,17)$. Therefore, the relevance of fluid administration has been studied for many years and it has been reported that dynamic measurements based on cardiopulmonary interactions in mechanically ventilated patients are the best predictors of fluid response (18). Stroke volume variation (SVV), pulse pressure variation (PPV), and PVI are among the most frequently used dynamic parameters in the management of perioperative fluid therapy.

PVI allows clinicians to determine fluid sensitivity in critically ill patients. SpHb allows continuous analysis of hemoglobin concentration and helps in making decisions about blood transfusions. It is especially important for operations where blood loss is high and time loss cannot be tolerated (19). The reliability of these parameters has been confirmed in intensive care patients and open abdominal cases in the operating room $(5,20,21)$. However, the effect of pneumoperitonium on these parameters in obese is not clear.

In fact, studies evaluating the effects of pneumoperitonium on non-invasive hemodynamic monitoring parameters have attracted attention in recent years. Hoiseth et al. (22) showed that PVI increased during pneumoperitonium in their studies in which they investigated the dynamic variables of fluid responsiveness in patients undergoing laparoscopic surgery. Liu et al. (3) showed that PVI increased with pneumoperitonium and that PVI value decreased to pre-pneumoperitonium values after desufflation. However, the number of studies investigating the effects of obesity is very limited. However, determination of intravascular volume is critical in obese patients. Because pneumoperitonium created for adequate visualization of the operative area in laparoscopic surgeries results in a high pressure increase in the abdomen. As with non-obese patients, intraabdominal pressure is adjusted up to $12-15 \mathrm{~mm} \mathrm{Hg}$ during laparoscopy in obese patients. Normal intra-abdominal pressure of non-obese individuals is $5 \mathrm{~mm} \mathrm{Hg}$ or less (23). In contrast, obese patients may have a chronically elevated intraabdominal pressure up to 9 to $10 \mathrm{~mm} \mathrm{Hg}$. (24). Increased intraabdominal pressure increases venous stasis, reduces portal venous blood flow, increases airway pressure and impairs heart function (25). To minimize these effects, it is necessary to optimize the intravascular volume to make appropriate ventilation adjustments, to use appropriate compression devices to minimize venous stasis, and to minimize the effects of increased intra-abdominal pressure on kidney and heart function. At this point, the importance of hemodynamic monitoring is obvious for intravascular volume optimization.

In this study, in which we examined the effects of pneumoperitoneum on non-invasive hemodynamic monitoring parameters, PVI and SpHb, we observed that PVI increased and SpHb decreased by abdominal insufflation in all patients. However, these changes were only significant in the obese patient group. DeBarros et al. (14) compared open surgery, laparoscopic obesity surgery and laparoscopic surgery in non-obese patients. In this study, they identified an incorrect increase in PVI with insufflation. This finding coincides with our study. In the same study, they observed a change on $\mathrm{SpHb}$ with insufflation, but this change was not statistically significant. The difference between our study and this study may be due to inclusion of different types of laparoscopic surgeries into their studies. Since we investigated the effects of obesity and pneumoperitonium in our study, we preferred laparoscopic cholecystectomy surgeries with minimal fluid losses and bleeding that may affect PVI and SpHb measurements, and a short operation duration of laparoscopic cholecystectomy surgeries. We tried to provide standardization by including the single type of surgery in our study. We also tried to minimize the effects of comorbid diseases by including only ASA 1-2 patients in our study. De barros et al. included different types of laparoscopic surgeries without ASA limitation in their studies. As a matter of fact, the average ASA level was stated as 3 in their studies. These situations may be the reason for the differences between the two studies. 
This study has some limitations. First of all, our study is an observational study with limited sample size. Second, we included only ASA 1-2 patients in our study. Because we wanted to evaluate only the effects of obesity and pneumoperitoneum in our study, we wanted to rule out PVI and SpHb changes that may occur due to different comorbid diseases. More different studies are needed with ASA3-4 patients. Finally, we preferred laparoscopic cholecystectomy operations to standardize patient groups. The amount of intraoperative bleeding was minimal and the need for blood transfusion did not occur in any patient. Further studies are needed to evaluate the effect of obesity and pneumoperitonium on noninvasive monitoring in large laparoscopic operations such as large oncological resections, thoracic and colorectal surgeries, that blood loss is predicted and require blood transfusion.

As a result, pneumoperitonium in obese can lead to errors in PVI and SpHb monitoring. Although these two parameters are very valuable in intraoperative follow-up, they should be used carefully during laparoscopic procedures in obese patients. If these parameters (PVI and $\mathrm{SpHb}$ ) are considered to be used in bariatric surgeries, which are the most common surgical procedure with the combination of obesity and pneumoperitonium, this information should be considered. According to our knowledge, this study is the first study with standardized patient groups on the reliability of PVI and SpHb of obesity and pneumoperitoneum. In order to evaluate the usefulness of non-invasive hemodynamic monitoring in laparoscopic procedures in obese, further research is needed in larger laparoscopic procedures.

\section{Funding}

The authors declare no funding sources of this present study

\section{Conflict of Interests}

The authors declare no conflict of interests

\section{References}

1. Himal HS. Minimally invasive (laparoscopic) surgery. Surg Endosc 2002;8:265-270. doi:10.1007/s00464001-8275-7

2. Freise H, Van Aken HK. Risks and benefits of thoracic epidural anaesthesia. BJA 2011; 107: 859-68. doi:10.1093/bja/aer339

3. Liu F, Zhu S, Ji Q, et all. The impact of intra-abdominal pressure on the stroke volume variation and plethysmographic variability index in patients undergoing laparoscopic cholecystectomy. BioScience Trends. 2015; 9(2):129-133. doi:10.5582/bst.2015.01029

4. Aykac ZZ, Arslantas MK. Fluid therapy and management (II) monitoring and prediction of fluid responsiveness. GKD Anest Yoğ Bak Dern Derg. 2018; 24(1): 1-10, doi: 10.5222/GKDAD.2018.001

5. Cannesson M, Desebbe O, Rosamel P, et al. Pleth variability index to monitor the respiratory variations in the pulse oximeter plethysmographic waveform amplitude and predict fluid responsiveness in the operating theatre. Br J Anaesth. 2008;101(2):200-206. doi: 10.1093/bja/aen133.

6. Pişkin Ö, Öz İ. Accuracy of pleth variability index compared with inferior vena cava diameter to predict fluid responsiveness in mechanically ventilated patients. Medicine (Baltimore). 2017; 96 (47):e8889. doi: $10.1097 / \mathrm{MD} .000000000000888$.

7. Gamal M, Abdelhamid B, Zakaria D, et all. Evaluation of Noninvasive Hemoglobin Monitoring in Trauma Patients with Low Hemoglobin Levels. Shock. 2018;49(2):150-153. doi: 10.1097/SHK.0000000000000949.

8. Chu H, Wang Y, Sun Y, et all. Accuracy of pleth variability index to predict fluid responsiveness in mechanically ventilated patients: a systematic review and meta-analysis. J Clin Monit Comput. 2016;30(3):265-274. doi: 10.1007/s10877-015-9742-3

9. Pinsky MR. Functional hemodynamic monitoring. Crit Care Clin. 2015;31(1):89-111. doi: 10.1016/j.ccc.2014.08.005

10. Henny CP, Hofland J. Laparoscopic surgery: pitfalls due to anesthesia, positioning, and pneumoperitoneum. Surg Endosc. 2005;19(9): 1163-1171. doi:10.1007/s00464-004-2250-z 
11. Balderi T, Forfori F, Marra V, Di Salvo C, Dorigo M, Anselmino M, Giunta F. Continuous hemodynamic monitoring during laparoscopic gastric bypass in superobese patients by pressure recording analytical method. Obesity surgery. 2008; 18(8), 1007-1014.

12. Marik PE, Baram M, Vahid B. Does central venous pressure predict fluid responsiveness? A systematic review of the literature and the tale of seven mares. Chest. 2008;134(1):172-178. doi: 10.1378/chest.072331.

13. Marik PE, Cavallazzi R, Vasu T, et all. Dynamic changes in arterial waveform derived variables and fluid responsiveness in mechanically ventilated patients: a systematic review of the literature. Crit Care Med. 2009;37(9):2642-7. doi: 10.1097/CCM.0b013e3181a590da.

14. DeBarros M, Causey MW, Chesley P, et all. Reliability of Continuous Non-Invasive Assessment of Hemoglobin and Fluid Responsiveness: Impact of Obesity and Abdominal Insufflation Pressures. Obes Surg. 2015;25(7):1142-1148. doi:10.1007/s11695-014-1505-6

15. Gustafsson UO, Hausel J, Thorell A, et all. Enhanced Recovery After Surgery Study Group. Adherence to the enhanced recovery after surgery protocol and outcomes after colorectal cancer surgery. Arch Surg. 2011;146:571-577. doi: 10.1001/archsurg.2010.309.

16. Zimmermann M, Feibicke T, Keyl C, et al. Accuracy of stroke volume variation compared with pleth variability index to predict fluid responsiveness inmechanically ventilated patients undergoing major surgery. Eur J Anaesthesiol. 2010;27:555-561. doi: 10.1097/EJA.0b013e328335fbd1.

17. Corcoran T, Rhodes JE, Clarke S, et all. Perioperative fluid management strategies in major surgery: a stratified meta-analysis. Anesth Analg. 2012;114:640-651. doi: 10.1213/ANE.0b013e318240d6eb.

18. Lopes MR, Oliveira MA, Pereira VO, et all. Goal-directed fluid management based on pulse pressure variation monitoring during high-risk surgery: a pilot randomized controlled trial. Crit Care. 2007;11:R100. doi: 10.1186/cc6117

19. Gupta N, Kulkarni A, Bhargava AK, et all. Utility of non-invasive haemoglobin monitoring in oncosurgery patients. Indian J Anaesth. 2017;61(7):543-548. doi:10.4103/ija.IJA_707_16

20. Causey MW, Miller S, Foster A, et all. Validation of noninvasive hemoglobin measurements using the masimo radical-7 SpHb station. Am J Surg. 2011;201(5):592-598. doi: 10.1016/j.amjsurg.2011.01.020.

21. Loupec T, Nanadoumgar H, Frasca D, et al. Pleth variability index predicts fluid responsiveness in critically ill patients. Crit Care Med. 2011;39(2):294-299. doi: 10.1097/CCM.0b013e3181ffde1c

22. Hoiseth LO, Hoff IE, Myre K, et all. Dynamic variables of fluid responsiveness during pneumoperitoneum and laparoscopic surgery. Acta Anaesthesiol Scand. 2012;56(6):777-786. doi: 10.1111/j.13996576.2011.02641.x

23. Sanchez NC, Tenofsky PL, Dort JM, et all. What is normal intraabdominal pressure? Am Surg. 2001;67:243-248.

24. Nguyen NT, Lee SL, Anderson JT, et all. Evaluation of intraabdominal pressure after open and laparoscopic gastric bypass. Obes Surg. 2001; 11:40-45. doi: 10.1381/096089201321454097

25. Nguyen NT, Wolfe BM. The physiologic effects of pneumoperitoneum in the morbidly obese. Ann Surg. 2005;241:219-226. doi: 10.1097/01.sla.0000151791.93571.70 


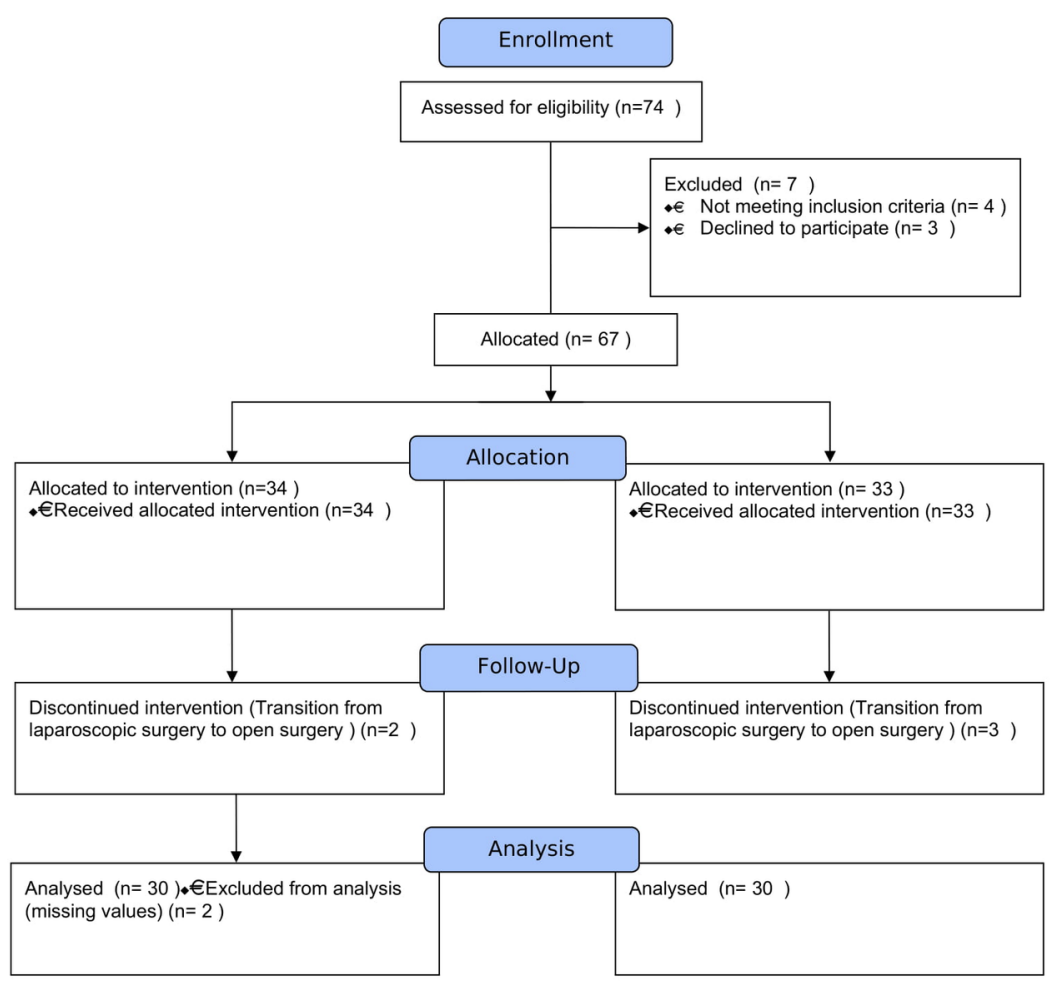




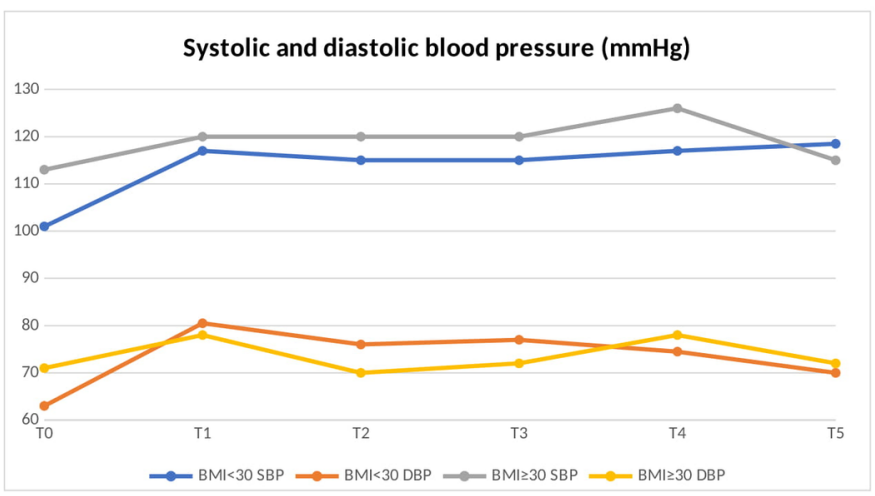




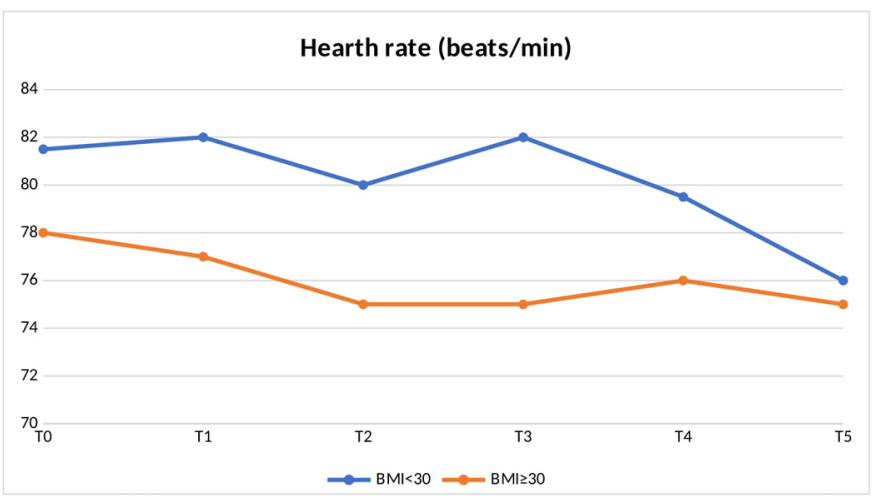



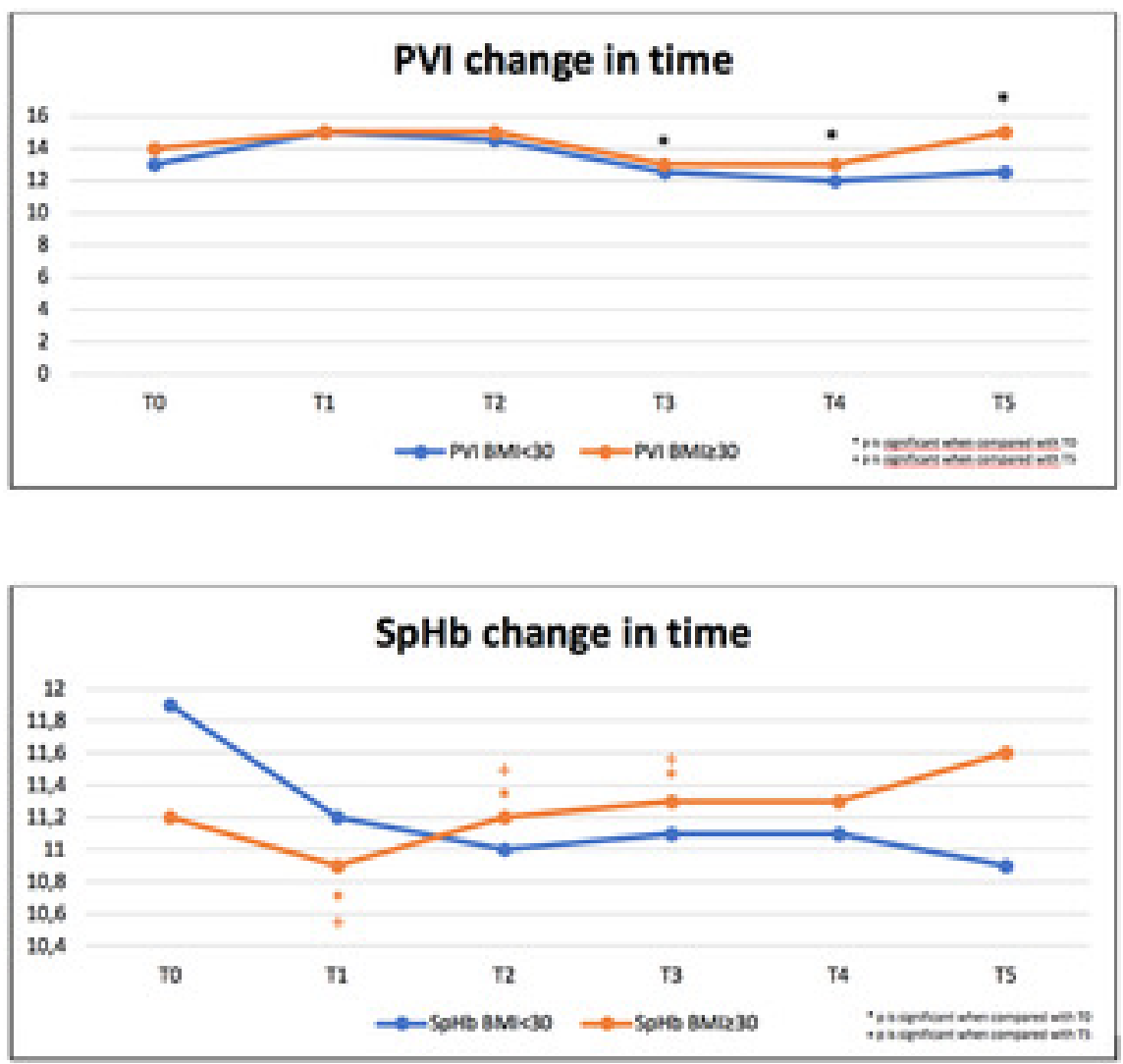

\section{Hosted file}

Table 1.docx available at https://authorea.com/users/398524/articles/526822-the-effectsof-body-weight-and-pneumoperitoneum-on-pleth-variability-index-and-total-hemoglobin-inpatients-undergoing-laparoscopic-cholecystectomy-a-prospective-observational-study

\section{Hosted file}

Table 2.docx available at https://authorea.com/users/398524/articles/526822-the-effectsof-body-weight-and-pneumoperitoneum-on-pleth-variability-index-and-total-hemoglobin-inpatients-undergoing-laparoscopic-cholecystectomy-a-prospective-observational-study 